\title{
In vivo skin imaging for hydration and micro relief-measurement
}

\author{
Kardosova Z, Hegyi V \\ Department of Children's Dermatovenerology, Children's University Hospital, Bratislava, Slovakia. \\ kohut.zuzana@gmail.com
}

\begin{abstract}
The aim: We present the results of our work with device used for measurement of skin capacitance before and after application of moisturizing creams and results of experiment performed on cellulose filter papers soaked with different solvents.

Methods and results: The measurements were performed by a device built on capacitance sensor, which provides an investigator with a capacitance image of the skin. The capacitance values are coded in a range of 256 gray levels then the skin hydration can be characterized using parameters derived from gray level histogram by specific software. The images obtained by device allow a highly precise observation of skin topography. Conclusion: Measuring of skin capacitance brings new, objective, reliable information about topographical, physical and chemical parameters of the skin. The study shows that there is a good correlation between the average grayscale values and skin hydration. In future works we need to complete more comparison studies, interpret the average grayscale values to skin hydration levels and use it for follow-up of dynamics of skin micro-relief and hydration changes (Fig. 6, Ref. 15). Full Text in PDF www.elis.sk.

Key words: skin, micro-relief, dielectrical properties, hydration, capacitance sensor.
\end{abstract}

Characterizing the skin hydration and its micro-relief has a high importance in the monitoring of dynamic changes in the course of chronic skin disorders as well as in the cosmetic science. For skin physiologists, this interface between the environment and the biological medium has several important function (barrier, mechanical, thermal, sensitive, etc) that, all together, justify the efforts undertaken during recent years by physicists and bioengineers in developing new techniques for measuring, in vivo, some physical properties of the skin (7). Various research laboratories have developed some few devices allowing measuring the electrical skin properties, all being based on the skin capacitance, conductance or impedance (5), with different kinds of electrodes, sensors and working by various measurement frequencies (3). One of them is the non optical skin capacitance imaging using sensors based on silicon technology primary developed to sensing fingerprints for security reasons $(2,7,11,13)$. The analysis paper proves a significant linear correlation between the results obtained by Corneometer device (taken into consideration as reference value for skin hydration) and the averaged parameters measured by SkinChip ${ }^{\circledR}(1,7,12)$.

\section{Apparatus}

The scientific laboratories L'Oréal (Paris, France) in cooperation with ST Microelectronics (Geneva, Switzerland) modified the chip technology for fingerprint sensor and developed a

Department of Children's Dermatovenerology, Children's University Hospital, Bratislava, Slovakia

Address for correspondence: Z. Kardosova, MD, Department of Children's Dermatovenerology, Children's University Hospital, Limbova 1 , SK-833 40 Bratislava, Slovakia. device enabling to get the image of skin surface - SkinChip ${ }^{\circledR}(1$, $6,7,8)$. On a similar principle, we modified the chip technology for fingerprint sensor (commercially manufactured as SPF200USB fingerprint sensor) and developed a device able to imaging skin surface which extends the existing diagnostic and therapeutic procedures in pathological conditions influencing micro-relief and skin hydration. Fingerprint sensor consists of a 256 column x 300 row array of tiny metal electrodes. Each electrode acts as one plate in a capacitor and the finger acts as the second plate in a capacitor. A passivation layer on the surface of the device forms a tough outer shell, protecting the device from abrasion, chemicals, moisture and other forms of damage. The SPF200-USB is manufactured in standard CMOS technology, a mature and cost effective manufacturing method (15). The measuring principle of our device is based on capacitance measurement of a dielectric medium. Any change in the dielectric constant due to skin surface hydration variation alters the capacitance of a precision measuring capacitor (4). The capacitance method treats the water content in the skin as a dielectric material. Thus, for a capacitance measuring device, the increase in capacitance is proportional to the quantity of water in the skin. The main advantage of using the capacitance method is its ability to produce results with a high accuracy (10). The capacitance is modulated primarily as a function of the distance between the capacitive sensor and the skin micro-relief (5). Device sensor actively scans the capacitance and records detailed skin images in resolution $50 \mu \mathrm{m} /$ pixel. After application of sensor on the skin, we obtain the image composed from 256 degrees of gray scale while black colour represents a very high capacitance and white colour a very low capacitance of skin in the examined area. This explains why the gray degrees of outgoing image are darker under keratinocytes than under skin wrinkles. 


\section{Material and methods}

The measurements were performed by a device built on capacitance sensor, which provides investigator with a capacitance image of the skin. The capacitance values are coded in a range of 256 gray levels, then the skin hydration can be characterized using parameters derived from gray level histogram by specific software. The moisturizing cream effect measurements were performed before and after application of cream on volar forearm of nine volunteers in 10 minutes intervals during 60 minutes. We used two different moisturizing creams: $1 \%$ lactacid in cremor anionicus and $1 \%$ lactacid in Synderman. A small amount of a moisturizing cream was applied on the skin and rubbed into $4 \times 4 \mathrm{~cm}^{2}$ area of the volar forearm skin. To reduce the error of skin humidity measurements, the pressure applied on the skin must be controlled. Figure 1 shows the sensor with a spring system. The spring system can offer a constant force of $20 \mathrm{~N}$ and is connected with a skin moisture sensor. The constant force reduces the error of measurement (5). All



Fig. 1. Schematic picture of probe for detecting the skin moisture with a spring system.
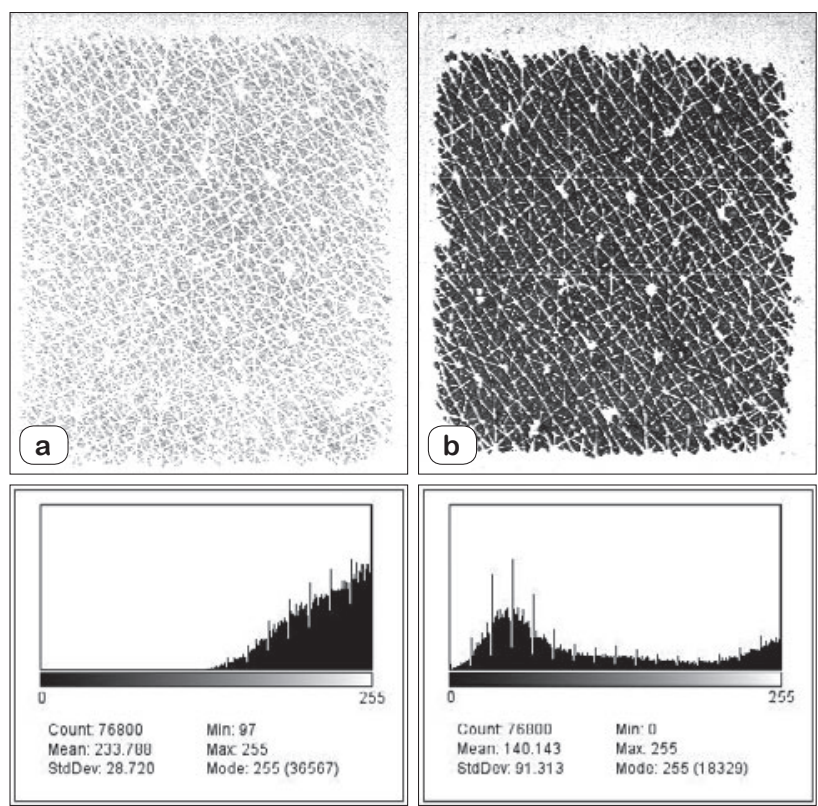

Fig. 2. Examples of capacitance images of the skin on volar side of the forearm and gray-level histograms, a) before and b) immediately after application of a moisturizing cream.

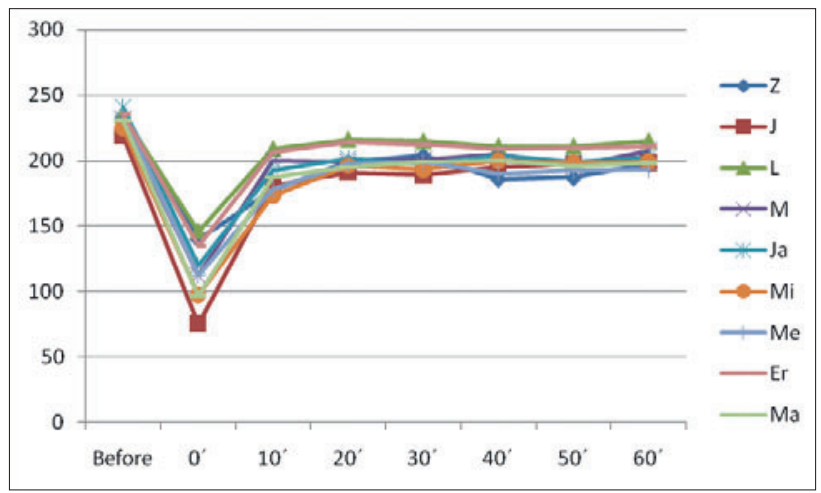

Fig. 3. The averaged grayscale values of the skin capacitance images in time during moisturizing cream effect measurement used $1 \%$ Lactacid in cremor anionicus.

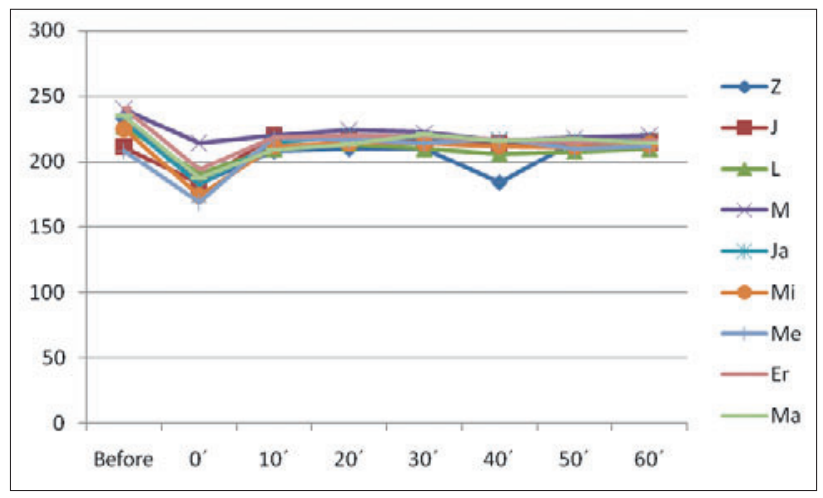

Fig. 4. The averaged grayscale values of the skin capacitance images in time during moisturizing cream effect measurement used $1 \%$ Lactacid in Synderman.

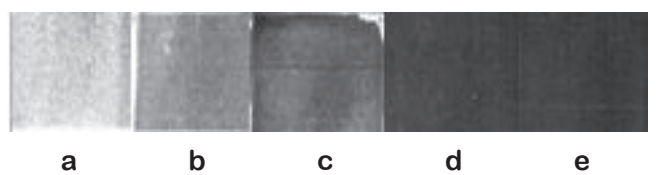

Fig. 5. Examples of capacitance of five different solvents. a) 2-ethyl1-hexanol, b) isopropanol, c) ethanol, d) water/ethanol, e) water.

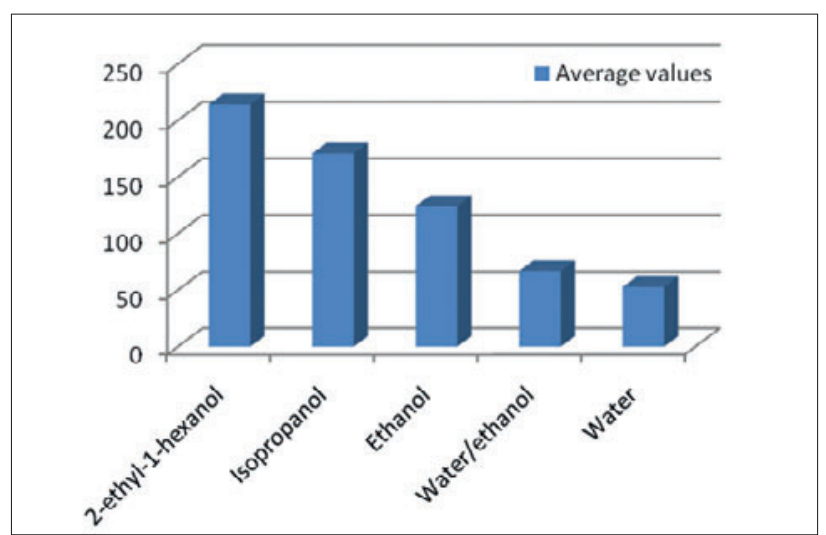

Fig. 6. The averaged grayscale values of capacitance of five different solvents. 
measurements were performed under normal ambient laboratory conditions of $20-25^{\circ} \mathrm{C}$ and $40-50$ RH. Calibration of our device was carried out on cellulose filter papers saturated with solutions at different dielectrical properties: 2-ethyl-1-Hexanol, Isopropanol 99 $\%$, Ethanol $90 \%$, Ethanol/Water 1:1 and Water. 10 measurements were performed for each solution. The sensor was pressed by the constant force of $20 \mathrm{~N}$ in 5 seconds interval. The capacitance for each solution is the mean value of 10 individual measurements.

\section{Results and discussion}

The images obtained by device allow a highly precise observation of skin topography. Figure 2 shows examples of capacitance images of the skin on volar side of the forearm and gray-level histograms before and immediately after application of a moisturizing cream. In observation of moisturizing cream effect measurements, the grayscale values decrease significantly immediately after application of the moisturizing cream, then as the cream starts disappearing from skin, the grayscale values increase back to their normal levels. Figures 3 and 4 show the averaged grayscale values of the skin hydration images during the moisturizing cream effect measurement, used $1 \%$ lactacid in Synderman and $1 \%$ lactacid in cremor anionicus. Sekiguchi et all reported on measurements with their sensor chip. They compared measuring results on normal skin, atopic (dry) skin and skin moisturized by a humectant. In their study the change of capacitance of skin treated with a humectant was much higher compared to skin not treated. In case of hydrated skin the measurements have been carried out 20 min after applying the humectant (8). Singh et al studied effect of moisturizing creams by a special device based on fingerprint sensor. In their work course of curve that represents the moisturizing cream effect is very similar to ours. The effect of their moisturizing cream lasts about 30 minutes (14). Calibration has brought some interesting results of capacitance: the lowest grayscale values of capacitance were measured using the water, then the values increase from a solution of ethanol with water to the highest one, solvent of 2-ethyl-1-hexanol. Figure 5 shows examples of capacitance images of five different solvents used in this measurement.

2-ethyl-1-hexanol represented very dry skin, isopropanol $99 \%$ dry skin, ethanol $90 \%$ normal to moisturized skin, ethanol/water 1:1 well-moisturized skin and water as a control test. Figure 6 shows averaged grayscale values of capacitance of five different solvents.

\section{Conclusion}

The skin capacitance imaging provides us with both visualisation and quantification of skin micro-relief and skin surface hydration in non-invasive way in vivo. The study offers our experience with a special device based on capacitive fingerprint sensor and a pilot measurements of skin hydration. In future works we need to complete more comparison studies, interpret the average grayscale values to skin hydration levels and use it for follow-up of dynamics of skin micro-relief and hydration changes.

\section{References}

1. Batisse D, Giron F, Lévequê JL. Capacitance imaging of the skin surface. Skin Res Technol 2006; 12: 99-104.

2. Bazin R, Laquiéze S, Rosillo A, Lévequê JL. Photoaging of the chest analyzed by capacitance imaging. Skin Res Technol 2010; 16: 23-29.

3. Darlenski R, Sassning S, Tsankov N, Fluhr JW. Non-invasive in vivomethods for investigation of the skin barrier physical properties. Eur J Pharm Biopharm 2009; 72: 295-303.

4. Huang Ting-Hsiang, Chou Jung-Chuan, Sun Tai-Ping, Hsiung ShenKan. A device for skin moisture and environment humidity detection. Sensors \& Actuators: B. Chemical 2008; 1: 206-212.

5. Gherardi A. A skin surface characterization system based on capacitive image analysis. $\mathrm{PhD}$ Thesis 2007. $\mathrm{PhD}$ program in Information technology.

6. Lévequê JL. Capacitance Imaging of the Skin Surface. In: Wilhelm KP, Elsner P, Berardesca E, Maibach HI. Bioengineering of the skin. Skin Imaging and Analysis. 2nd edn. New York: Informa Healthcare, 2007: 331-337.

7. Lévequê JL, Querleux B. SkinChip ${ }$, a new tool for investigating the skin surface in vivo. Skin Res Technol 2003; 9: 343-347.

8. Piérard GE, Lévequê JL. What is SkinChip ${ }^{\circledR}$ ? From silicon image sensor technology to SkinChip ${ }^{\circledR}$. Dermatology 2004; 208: 291-292.

9. Sekiguchi N, Komeda T, Funakubo H, Chabicovsky R, Nicolics J, Stangl G. Micro-Sensor for the Measurement of water content in the human skin, Sensors and Actuators 2001, B 78: 326-330.

10. Truong S. Design of a handheld skin moisture measuring device for application towards eczema. EE 4BI6 Electrical Engineering Biomedical Capstones. 2009, paper 15.

11. Uhoda E, Lévequê JL, Piérard GE. Silicon mage sensor technology for in vivo detection of surfactant-induced corneocyte swelling and drying. Dermatology 2005; 210:184-188.

12. Xhauflaire-Uhoda E, Loussouarn G, Haubrechts Ch, Léger DS, Piérard GE. Skin capacitance imaging and corneosurfametry. A comparative assessment of the impact of surfactants on stratum corneum. Contact Dermatitis 2006; 54: 249-53.

13. Xhauflaire-Uhoda E, Piérard GE, Quantresooz P. The skin landscape following nonoptical capacitance imaging. Am J Clin Dermatol 2010; 11 (2): 89-94.

14. http://ecce1.lsbu.ac.uk/research/photo/PX-SC5-poster.pdf

15. http://www.fujitsu.com/downloads/MICRO/fmal/biometricsensor/ spf200factsheet.pdf 